\title{
Maternal protein restriction induces alterations in insulin signaling and ATP sensitive potassium channel protein in hypothalami of intrauterine growth restriction fetal rats
}

\author{
Xiaomei Liu, ${ }^{1, *}$ Ying $\mathrm{Qi}^{,}{ }^{2}$ Hong Gao, ${ }^{1}$ Yisheng Jiao, ${ }^{3} \mathrm{Hui}$ Gu, ${ }^{1}$ Jianing Miao' ${ }^{1}$ and Zhengwei Yuan ${ }^{1}$ \\ ${ }^{1}$ Key Laboratory of Health Ministry for Congenital Malformations, ${ }^{2}$ Virus Laboratory and ${ }^{3}$ Department of Obstetrics and Gynecology, \\ Shengjing Hospital of China Medical University, Shenyang, 110004, China
}

(Received 25 February, 2012; Accepted 20 June, 2012; Published online 20 November, 2012)

\begin{abstract}
It is well recognized that intrauterine growth restriction leads to the development of insulin resistance and type 2 diabetes mellitus in adulthood. To investigate the mechanisms behind this "metabolic imprinting" phenomenon, we examined the impact of maternal undernutrition on insulin signaling pathway and the ATP sensitive potassium channel expression in the hypothalamus of intrauterine growth restriction fetus. Intrauterine growth restriction rat model was developed through maternal low protein diet. The expression and activated levels of insulin signaling molecules and $K_{A T P}$ protein in the hypothalami which were dissected at 20 days of gestation, were analyzed by western blot and real time PCR. The tyrosine phosphorylation levels of the insulin receptor substrate 2 and phosphatidylinositol 3'-kinase p85 $\alpha$ in the hypothalami of intrauterine growth restriction fetus were markedly reduced. There was also a downregulation of the hypothalamic ATP sensitive potassium channel subunit, sulfonylurea receptor 1, which conveys the insulin signaling. Moreover, the abundances of gluconeogenesis enzymes were increased in the intrauterine growth restriction livers, though no correlation was observed between sulfonylurea receptor 1 and gluconeogenesis enzymes. Our data suggested that aberrant intrauterine milieu impaired insulin signaling in the hypothalamus, and these alterations early in life might contribute to the predisposition of the intrauterine growth restriction fetus toward the adult metabolic disorders.
\end{abstract}

Key Words: perinatal nutrition, hypothalamus, insulin signaling, ATP sensitive potassium channel

Ttrauterine growth restriction (IUGR) affects $3-10 \%$ of pregnancies, and is recognized as a major cause of fetal and neonatal morbidity and mortality. ${ }^{(1)}$ Epidemiological and experimental studies indicate that IUGR newborns are strongly predisposed to the development of metabolic syndrome consisting of insulin resistance, type 2 diabetes mellitus, obesity, hypertension, and coronary artery disease in later life. ${ }^{(2-5)}$ We developed an IUGR model in rats by maternal protein restriction, whereby the animals developed glucose intolerance and insulin resistance in adulthood. ${ }^{(6)}$ Our previous results showed that hepatic insulin signaling was disturbed in undernutrition offsprings with this animal model, in line with other studies on the insulin target tissues (liver, muscle and adipose) from IUGR animals. ${ }^{(7-10)}$

Defects in the insulin-signaling cascade have been implicated in the pathogenesis of insulin resistance. Insulin first binds to the $\alpha$ subunit of the insulin receptor (IR), and this leads to activation of cascade of events which begin with activation of the IR, followed by tyrosine phosphorylation of insulin receptor substrate (IRS), which induces the activation of downstream pathways such as the phosphatidylinositol 3'-kinase (PI3K). The effect of IUGR on insulin signaling has been studied mainly in classic insulin target tissues, seldomly in the central nervous system (CNS) which was assumed to be insulin insensitive tissue. Over the past few years it has become clear that insulin had profound effects in the CNS. Studies performed on rodents have defined insulin action in the $\mathrm{CNS}$ as a critical determinant of energy homeostasis and of peripheral glucose metabolism. ${ }^{(11)}$ Insulin modifies peripheral glucose metabolism through IR localized in the hypothalamus, especially liver glucose synthesis. Third-cerebral-ventricle administration of insulin increases insulin sensitivity in peripheral tissues and the improvement in peripheral insulin action is associated with a reduction in hepatic glucose production. ${ }^{(12)}$ The hypothalamic insulin signal is conveyed to the motor nucleus of the vagus by ATP-sensitive potassium (KATP) channel and reaches the liver via vagal efferent fibers (Fig. 1). ${ }^{(13)}$ The K $\mathrm{K}_{\text {ATP }}$ channels in the hypothalamus are heteromultimers composed of two subunits: a regulatory sulfonylurea receptor (SUR), which contains two adenine nucleotide binding domains, and a pore-forming inward rectifying potassium channel (Kir6.x), which conducts potassium ions. ${ }^{(14)}$ Mutations in the SUR1 and Kir6.x are known to cause a group of congenital hyperinsulinemic disorders of humans collectively known as persistent hypoglycemic hyperinsulinism of infancy. ${ }^{(15)}$

The present study investigated the impact of IUGR, obtained through maternal protein restriction, on the expression of hypothalamic K KTP channels and key molecules of insulin signaling pathway. This work aimed to explore how maternal undernutrition influenced the development and function of the hypothalamus to regulate energy expenditure along with peripheral controlling factors.

\section{Materials and Methods}

Animals. Outbred Wistar rats of 10-12 weeks of age (230 $260 \mathrm{~g}$ ) were purchased from animal center of China Medical University. Female rats were mated overnight with a male. The morning that conception was verified by the presence of sperm in a vaginal smear was designated gestational day 0 . All animals were individually housed under standard conditions, and food

*To whom correspondence should be addressed. E-mail: liuxm1@sj-hospital.org 


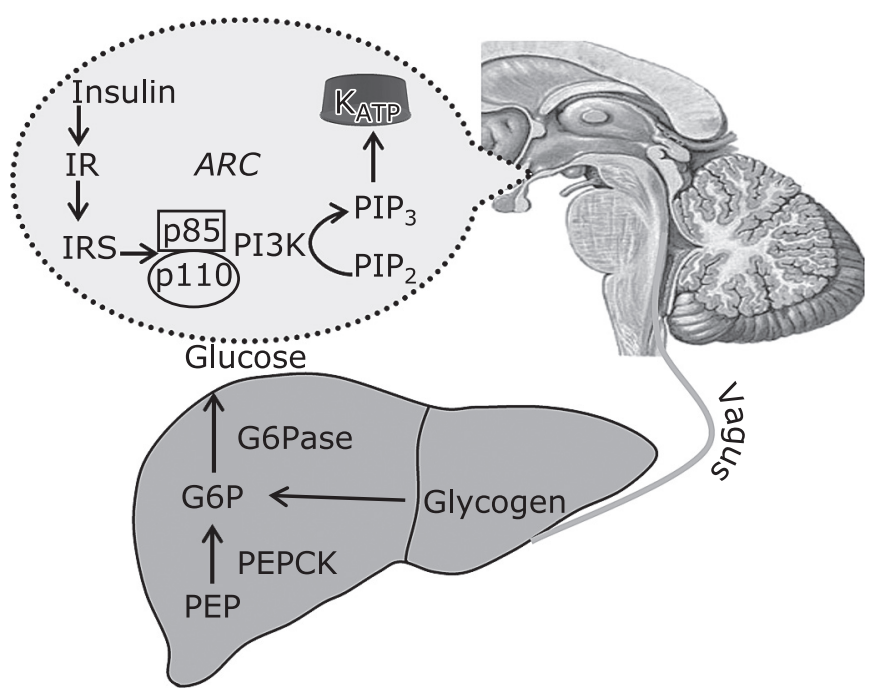

Fig. 1. Mechanism of insulin action in the hypothalamus arcuate nucleus (ARC). Insulin first binds to the insulin receptor (IR); this initiates a signal transduction cascade which begin with the activation of IR and insulin receptor substrate (IRS). Phosphotyrosines on the IRS proteins bind the p85 regulatory subunit of phosphatidylinositol 3'-kinase (PI3K), followed by phosphorylation of phosphatidylinositol 3,4-diphosphate $\left(\mathrm{PIP}_{2}\right)$ to phosphatidylinositol 3,4,5-triphosphate $\left(\mathrm{PIP}_{3}\right)$, which opens $\mathrm{K}_{\mathrm{ATP}}$ channels, thereby producing an outward flow of $\mathrm{K}^{+}$ions. This leads to hyperpolarization and reduced activity of the neuron. The hypothalamic insulin signal is conveyed to the motor nucleus of the vagus and reaches the liver via vagal efferent fibers. and water were provided ad libitum throughout the study. Control animals $(n=9)$ were maintained on standard chow $(20 \%$ protein) during gestation. The undernourished animals $(n=9)$ received an isocaloric low-protein diet ( $8 \%$ protein) as we described previously. ${ }^{(6)}$ Diets were purchased from Institute of Zoology, Chinese Academy of Sciences. At 20 days of gestation (term 21 days) pups were delivered by caesarian section and decapitated. IUGR referred to the fetus whose birth weight was two standard deviations less than the mean birth weight of the control group. Fetal blood was pooled (three or more) from the control or IUGR fetuses in a litter to quantify plasma insulin and glucose levels. Hypothalami were dissected, snap frozen and stored at $-80^{\circ} \mathrm{C}$ until use. Livers were harvested quickly and kept in the same way. All procedures were approved by the Animal Research Committee of China Medical University.

Analytic procedures. Blood glucose was determined in the local clinical laboratory. Liver glycogen concentration was measured by colorimetry assay using a liver glycogen test kit (Jiancheng, Nanjing, China) and the result was expressed as milligrams glycogen per gram wet weight liver tissue. Plasma insulin concentration was analyzed in duplicate by enzyme linked immunosorbent assay (Mercodia AB, Uppsala, Sweden) according to the manufacturer's protocol.

RNA isolation and real time PCR. Total RNA was extracted from snap-frozen hypothalamus using TRIzol reagent (Invitrogen, Carlsbad, CA) according to the manufacturer's protocol. All RNA samples were treated with DNase I following the manufacturer's protocol (M0303S, NEB, Toronto, ON) and quantified in duplicate using UV absorbance at $260 \mathrm{~nm}$. $1 \mu \mathrm{g}$ of total RNA was reversetranscribed into cDNA using the TaKaRa RNA PCR kit (DRR037, TaKaRa, Dalian, China) following the manufacturer's protocol. To minimize variation in the reverse transcription reaction, all

Table 1. Primers for real time reverse transcription polymerase chain reaction

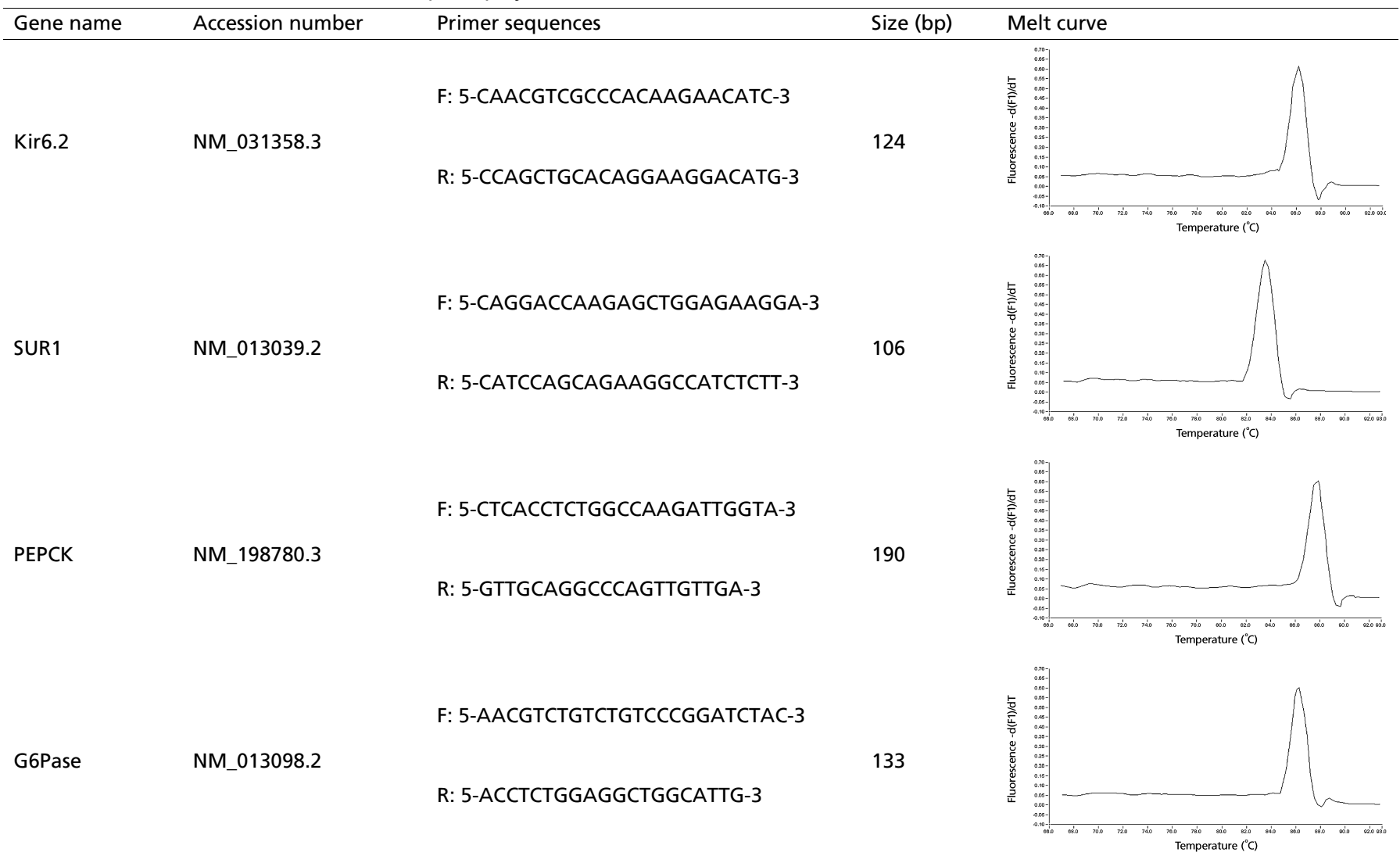


RNA samples from a single experimental setup were reverse transcribed simultaneously. Real time PCR was performed on LightCycler (Roche, Basel, Switzerland) using the primers described in Table 1. Control group values were used as calibrator. The relative level of mRNA expression was calculated using the $2^{-\Delta \Delta \mathrm{Ct}}$ method after normalization with $\beta$-actin (DR3783, TaKaRa) as a housekeeping gene. ${ }^{(16)}$

Protein lysate preparations. Subcellular fractionation of tissue proteins were fractionated into cytosolic and membrane pools using the Compartment Protein Extraction Kit (2145, Chemicon, Temecula, CA). In brief, different specimens (hypothalami and livers) were homogenized with lysis buffer containing sodium orthovanadate and cocktail of protease inhibitors, and centrifuged at $18,000 \mathrm{~g}$ at $4^{\circ} \mathrm{C}$ for $20 \mathrm{~min}$. The supernatant containing cytoplasmic proteins was saved. The pellet was redissolved in lysis buffer containing sodium deoxycholate, tergitol-type NP-40, sodium orthovanadate and cocktail of protease inhibitors, and centrifuged at $18,000 \mathrm{~g}$ at $4^{\circ} \mathrm{C}$ for $20 \mathrm{~min}$. The supernatant containing membrane proteins was saved. The protein concentration was determined using Protein Quantitative Detection Kit (BioRad, Hercules, CA).

Immunoblotting. Antibodies to Kir6.2, IR $\alpha$, IRS2, p $85 \alpha$, SUR1, phosphoenolpyruvate carboxykinase (PEPCK), and Glucose 6-phosphatase (G6Pase) were purchased from Santa Cruz Biotechnology (Santa Cruz, CA). Antibodies to phosphotyrosine PY20 and $\beta$-actin were from Abcam (Cambridge, UK). PhosphoIRS2 (Ser731) antibody was from Lifespan Biosciences (Seattle, WA). Equal amounts of protein were separated by electrophoresis on sodium dodecyl sulfate (SDS)-polyacrylamide gel and transfered onto a polyvinylidene difluoride (PVDF) membrane (Millipore, Billerica, MA) as described by Towbin et al. ${ }^{(17)}$ Non-specific binding sites were blocked by $2 \mathrm{~h}$ incubation with $3 \%$ bovine serum album. The PVDF membrane with membrane fractions was immunoprobed with the anti-Kir6.2 and anti-IR $\alpha$ antibody at $4{ }^{\circ} \mathrm{C}$ overnight. The membrane with cytoplasmic fractions was probed with antibodies against IRS2, p-Ser731-IRS2, p85 $\alpha$, SUR1, PEPCK or G6Pase respectively. Blot was then exposed to horseradish peroxidase-conjugated secondary antibody and visualized using enhanced chemiluminescence reagents (GE Healthcare, Buckinghamshire, UK). An anti- $\beta$-actin antibody was used for loading control. All immunoblots were run at least in triplicate. Quantity One software (Bio-Rad) was applied for the analysis of the optical density of the protein bands. The relative expression quantity was illustrated as the percentage of the optical density (OD) of target protein, adjusted with the corresponding $\beta$-actin OD versus that of the normal control.

Immunoprecipitation. Tyrosine phospholation of IRS2 and p $85 \alpha$ was determined by immunoprecipitation with anti-phosphotyrosine antibody and then immunoblotted with anti-IRS2 and anti-p85 $\alpha$ antibody. Briefly, cytoplasm proteins were then incubated for $4 \mathrm{~h}$ at $4^{\circ} \mathrm{C}$ with anti-phosphotyrosine PY20 antibody. Then $25 \mu \mathrm{l}$ of Protein G-Agarose was added and rotated for $2 \mathrm{~h}$ at $4^{\circ} \mathrm{C}$. The immune complexes were centrifuged at $1,000 \mathrm{~g}$ at $4^{\circ} \mathrm{C}$ for $5 \mathrm{~min}$, after which, they were washed, and then eluted by boiling in SDS sample buffer. Eluted proteins were then resolved, transferred and probed with anti-IRS 2 or anti-p85 $\alpha$ as mentioned above.

Statistical analysis. All values are presented as the mean percent of control \pm standard error (SE). Comparisons between groups were performed by Student's two-tailed $t$ test. Pearson's correlation analysis was used to correlate SUR1 mRNA levels with PEPCK and G6Pase mRNA levels respectively. Statistical analysis was performed using SPSS17.0 (SPSS, Chicago, IL). $p<0.05$ was considered significant.

\section{Results}

Weight and metabolic profile. There was no difference in
Table 2. Fetal body weight, brain weight and metabolic profile $($ mean $\pm \mathrm{SE})$

\begin{tabular}{lcc}
\hline Group & Control & Undernourished \\
\hline Live birth & 104 & 98 \\
Fetal body weight $(\mathrm{g})$ & $3.973 \pm 0.43$ & $3.176 \pm 0.54^{* *}$ \\
Brain weight $(\mathrm{g})$ & $0.181 \pm 0.017$ & $0.153 \pm 0.012^{* *}$ \\
Brain/body $(\%)$ & $4.56 \pm 0.39$ & $4.82 \pm 0.5^{* *}$ \\
Fetus length $(\mathrm{mm})$ & $36.52 \pm 2.84$ & $33.47 \pm 2.16^{*}$ \\
Tail length (mm) & $3.837 \pm 0.49$ & $3.067 \pm 0.34^{*}$ \\
Fetal glucose (mmol/L) & $3.42 \pm 0.67$ & $2.75 \pm 0.61^{*}$ \\
Fetal glycogen (mg/g liver) & $9.28 \pm 1.34$ & $8.42 \pm 0.85$ \\
Fetal insulin (mU/L) & $94 \pm 8$ & $76 \pm 7^{*}$ \\
\hline
\end{tabular}

${ }^{*} p<0.05 ;{ }^{* *} p<0.01$ vs control.

the food intaken daily and the average litter size ( $10 \pm 3$ vs $11 \pm 2)$. Maternal protein restriction resulted in fetal growth restriction, as evidenced by a lower body weight (Table 2 ) and significantly higher incidence rate of IUGR (Undernourished: $56.12 \%$ vs control: $3.85 \%, p<0.001)$. Undernourished fetus also showed a significant reduction in brain weight, but the brain/body ratio increased $(p<0.01)$. There was no difference in the hepatic glycogen values between the two groups, whereas the concentrations of blood glucose and plasma insulin were remarkably lower in IUGR fetuses $(p<0.05)$ (Table 2).

Expression and activation of insulin signaling molecules in the hypothalamic. The expressions and activation of insulin signaling molecules in hypothalami were assessed by immunoblotting assay (Fig. 2). IR $\alpha$ expression had a tendency to be lower in hypothalami from IUGR fetuses, but the change did not achieve statistical significance. The total protein expressions of IRS2 and PI3K regulatory subunit $p 85 \alpha$ were unchanged in the IUGR hypothalami, nor was the level of serine phosphorylation of IRS2. However the levels of tyrosine phosphorylation of IRS2 and p $85 \alpha$ were significantly reduced by $52 \%$ and $27 \%$ respectively in the hypothalami of IUGR fetuses $(p<0.05)$.

Expression of KATP channels in the hypothalamic. According to real time PCR analysis, maternal undernutrition significantly $(p<0.05)$ downregulated the SUR1 mRNA levels in the hypothalami, whereas no significant differences were observed in the levels of Kir6.2 mRNA between IUGR and control rats (Fig. 3A). To obtain further information of the difference of proteins expression, we measured the protein levels of Kir6.2 and SUR1 using immunoblotting assay. In general, mRNA and protein levels of KATP channel were comparable. SUR1 protein expressions were downregulated by $30 \%$ in the IUGR rats $(p<0.05)$, and the Kir6.2 protein expressions did not differ between the two groups (Fig. $3 \mathrm{~B}$ and $\mathrm{C}$ ).

Expression of gluconeogenesis enzymes in the livers. Our previous work showed a marked increase in the expressions of PEPCK and G6Pase in the livers of IUGR rats at age 1 and 12 weeks. $^{(6)}$ To extend this study, we measured PEPCK and G6Pase expressions in fetal livers. PEPCK and G6Pase mRNA levels were upregulated by 2.9 and 1.6-fold respectively in IUGR compared with control livers $(p<0.05$, Fig. $4 \mathrm{~A})$. Consistent with the PCR data, striking differences were also found between IUGR and control rats in the relative expression ratios of PEPCK and G6Pase protein ( $p<0.05$, Fig. $4 \mathrm{~B}$ and $\mathrm{C})$.

Pearson's correlation analysis revealed that no correlation was observed between the levels of SUR 1 mRNA and those of PEPCK and G6Pase ( $r=0.210,0.153$ respectively).

\section{Discussion}

Maternal malnutrition during pregnancy obviously leads to IUGR because of inadequate nutrient delivery to the fetus. Our 

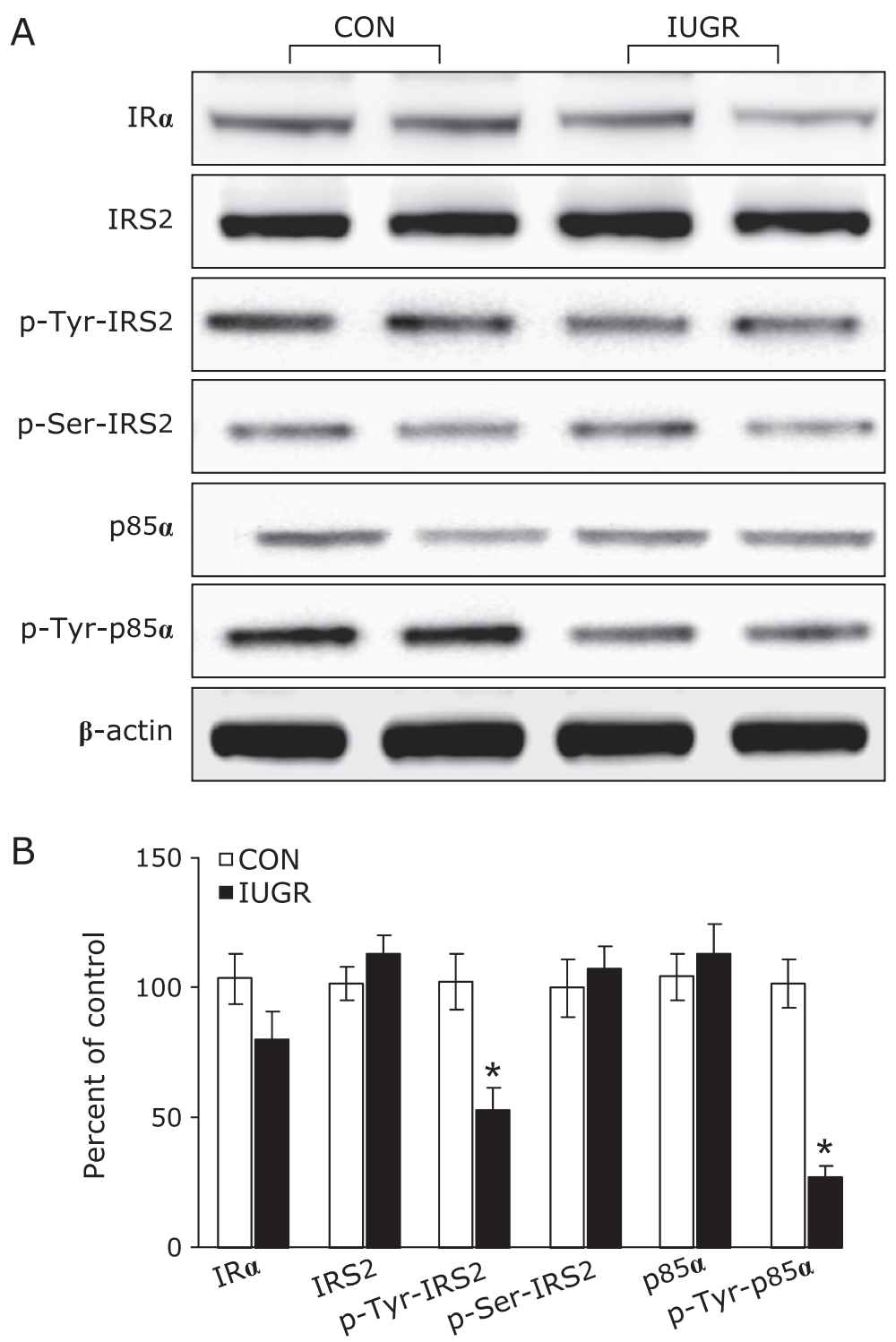

Fig. 2. The effect of maternal protein restriction on hypothalamic insulin signaling molecules. Representative immunoblots (A) and densitometric analyses (B) of insulin signaling proteins in hypothalami from control (white bars) and IUGR fetuses (black bars). CON, control; IUGR, intrauterine growth restriction. The data were normalized to the control, and represent the mean values $( \pm \mathrm{SE}), n=6$ per group. ${ }^{*} p<0.05$ vs control.

study showed that maternal undernutrition resulted in decreased brain weight but enhanced brain/body weight ratio in near term fetus, which indicated a partial preservation of brain development as compared with the peripheral organs. This well-known effect of brain sparing is usually considered an adaptation to protect the brain from more severe damage, at the expense of visceral organs. IUGR fetuses were hypoglycemic and hypoinsulinemia, in line with other reports and the phenotype of human IUGR newborns. ${ }^{(18,19)}$

Early undernutrition is known to increase risk of insulin resistance and diabetes in adulthood. ${ }^{(20)}$ Insulin resistance is associated with the post receptor defects in the intracellular insulin signaling cascade, leading to the failure of insulin to suppress hepatic glucose production. ${ }^{(19,21,22)}$ Previous research reported a series of changes in the insulin receptor signal transduction in the classic target tissues (liver, muscle and adipose) of IUGR human and animals. ${ }^{(23-26)}$ Besides modulating insulin function in peripheral organs, PI3K pathway has also been shown to control peripheral glucose metabolism by binding to the regulatory subunit SUR of KATP channels in the brain. ${ }^{(27,28)} \mathrm{K}_{\text {ATP }}$ channels are widely expressed in the brain: glia cells and neurons of the hypothalamus and the hippocampus. ${ }^{(29,30)}$ Insulin can activate the $\mathrm{K}_{\text {ATP }}$ channel which allows potassium ion flow from the cells, thus leads to cell hyperpolarization and decrease excitability of the neuron. This effect inhibits the gluconeogenesis in the liver finally through the vagus nerve. ${ }^{(31)}$ Studies demonstrated that activation of either insulin signaling or of $\mathrm{K}_{\text {ATP }}$ channels within the hypothalamus was sufficient to decrease blood glucose levels via a substantial inhibition of glucose production, which was largely due to a marked decrease in the hepatic abundance of PEPCK and G6Pase expression and of gluconeogenesis in the liver. Conversely, blockade of either insulin signaling or of KATP channels within the hypothalamus negated the effects of central insulin and halved the effects of systemic insulin on hepatic glucose production. ${ }^{(11,32)}$

The present study showed, for the first time, that the tyrosine phosphorylation levels of IRS 2 and PI3K p $85 \alpha$ were significantly impaired in the hypothalami of IUGR rats, which might block the insulin signaling transduction in hypothalamus. There was no 

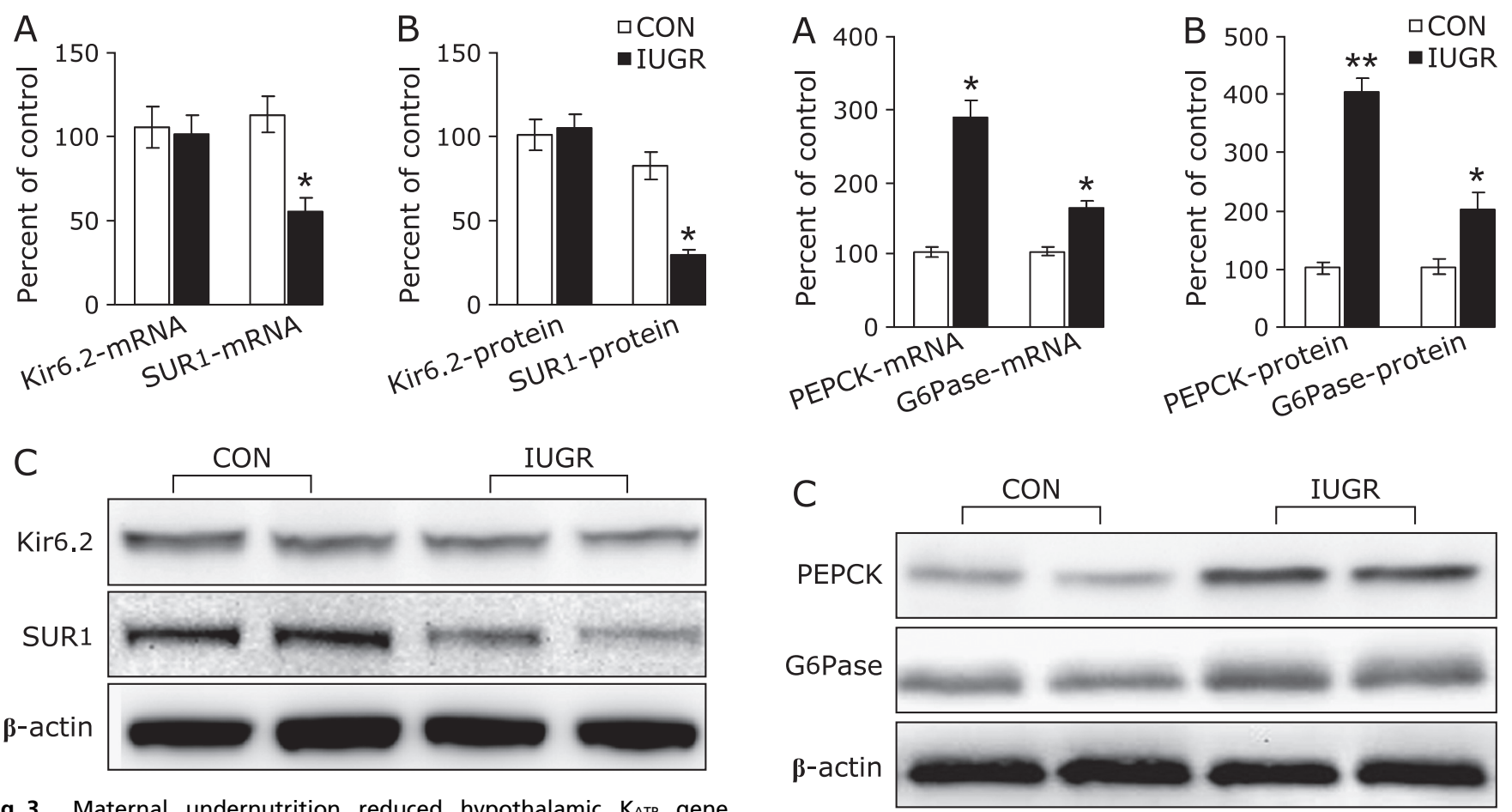

Fig. 3. Maternal undernutrition reduced hypothalamic KATP gene expression in fetuses. (A) Real time PCR analysis of KATP channel mRNA level in control (white bars) and IUGR (black bars) hypothalami. (B and C) Immunoblotting assay of KATP channel protein expression and representative immunoblots. The data were normalized to the control, and represent the mean values $( \pm \mathrm{SE}), n=6$ per group. ${ }^{\star} p<0.05$ vs control.

change in the expression and phosphorylation level of IR, in line with the observations in asymmetric IUGR fetal brain in Cited1 mutant mice. ${ }^{(33)}$ The results also indicated that maternal proteinrestriction caused the reduced expression of SUR1 in the fetal hypothalamus. In SUR1 null mice, insulin's ability to suppress hepatic gluconeogenesis was impaired. ${ }^{(34)}$ These results indicated that maternal protein restriction might interfere with insulin signaling transduction in hypothalamus and therefore influence the liver glucogenesis.

The other major finding of the present study was the upregulated expressions of PEPCK and G6Pase in the IUGR fetal livers. PEPCK and G6Pase are major rate-limiting enzymes in gluconeogenesis. Overexpression of either G6Pase or PEPCK has resulted in an elevation of hepatic glucose production and plasma glucose levels. ${ }^{(35)}$ The IUGR fetuses were hypoglycemic despite of the increased expression of gluconeogenesis enzymes, for prenatal gluconeogenesis is negligible as the fetus depends on maternal blood glucose as the source of energy and glycogen storage. The increased expression of gluconeogenesis enzymes in fetus might persist to adulthood, lead to augmented hepatic glucose production and increasing plasma glucose level in IUGR adults eventually. Previous studies have certified that PEPCK and G6Pase expressions were increased in offspring of protein-restricted, alcoholexposed and bilateral uterine artery ligation dams from childhood to adulthood. ${ }^{(7,35,36)}$ Contrary to our supposition, no correlation was observed between the expression of hypothalamic SUR1 and of hepatic PEPCK and G6Pase. Lane et al., ${ }^{(35)}$ reported that PEPCK and G6Pase expression correlated with the expression of peroxisome proliferator-activated receptor $\gamma$ coactivator- 1 in the livers of IUGR rats. Nevertheless, our results couldn't preclude the association between changes of hepatic gluconeogenesis enzymes and hypothalamic KATP channels in IUGR rats, since the concrete electrophysiological change of $\mathrm{K}_{\mathrm{ATP}}$ channels was unknown.

Fig. 4. Maternal undernutrition increased hepatic gluconeogenesis enzymes expression in fetuses. (A) Real time PCR analysis of phosphoenolpyruvate carboxykinase (PEPCK) and glucose 6-phosphatase (G6Pase) mRNA level in control (white bars) and IUGR (black bars) livers. (B and C) Immunoblotting assay of PEPCK and G6Pase protein expression and representative immunoblots. The data were normalized to the control, and represent the mean values ( $\pm \mathrm{SE}$ ), $n=6$ rats/group. ${ }^{*} p<0.05 ;{ }^{* *} p<0.01$ vs control.

Besides increasing liver glucogenesis, inhibition of insulin signaling in the brain produces an orexigenic effect, which resulted in a gain in body weight associated with peripheral insulin resistance. ${ }^{(31,37,38)}$ It was reported that adult offspring of maternal protein restriction was hyperphagia which was driven by a strong upregulation of hypothalamic orexigenic peptide neusopeptide $\mathrm{Y}$ (NPY) accompanied with low insulin level. ${ }^{(39)}$ SUR1-containing $\mathrm{K}_{\text {ATP }}$ channels are expressed in the NPY-expressing neurons; thence the relationship between NPY expression and the insulin signaling in the IUGR hypothalamus deserves further investigation.

The present study was limited to fetus, so from the data we couldn't know whether the changes in hypothalamus are transient or permanent. Moreover we couldn't investigate the effect of IUGR on the functional properties of hypothalamic KATP channel without the patch clamp.

In summary our data suggested that early undernutrition affected markedly the expression and/or activation level of key factors involved in insulin signaling transduction in the hypothalamus. These signaling transduction and enzymatic alterations induced by intrauterine malnutrition might contribute to the development of adult metabolic disorders.

\section{Acknowledgments}

This work was supported in part by grant (No. 81100432) from the National Natural Science Foundation of China. The authors would like to thank Lian Ji for her excellent technical assistance on animal anatomy. 


\section{Abbreviations}

CNS central nervous system

G6Pase glucose-6-phosphatase

IR insulin receptor

IRS insulin receptor substrate

IUGR intrauterine growth restriction

KATP channel ATP-sensitive potassium channel
Kir6.2 inward rectifying potassium channel 6.2

PEPCK phosphoenolpyruvate carboxykinase

PI3K phosphatidylinositol 3'-kinase

SUR sulfonylurea receptor

\section{Conflict of Interest}

No potential conflicts of interest were disclosed.

\section{References}

1 Miller J, Turan S, Baschat AA. Fetal growth restriction. Semin Perinatol 2008; 32: 274-280.

2 Benediktsson R, Lindsay RS, Noble J, Seckl JR, Edwards CR. Glucocorticoid exposure in utero: new model for adult hypertension. Lancet 1993; 341: 339341.

3 Barker DJ, Hales CN, Fall CH, Osmond C, Phipps K, Clark PM. Type 2 (non-insulin-dependent) diabetes mellitus, hypertension and hyperlipidaemia (syndrome X): relation to reduced fetal growth. Diabetologia 1993; 36: 6267.

4 Gluckman PD, Hanson MA, Pinal C. The developmental origins of adult disease. Matern Child Nutr 2005; 1: 130-141.

5 Cottrell EC, Ozanne SE. Developmental programming of energy balance and the metabolic syndrome. Proc Nutr Soc 2007; 66: 198-206.

6 Liu XM, Kong J, Song WW, Lu Y. Glucose metabolic and gluconeogenic pathways disturbance in the intrauterine growth restricted adult male rats. Chin Med Sci J 2009; 24: 208-212.

7 Vuguin P, Raab E, Liu B, Barzilai N, Simmons R. Hepatic insulin resistance precedes the development of diabetes in a model of intrauterine growth retardation. Diabetes 2004; 53: 2617-2622.

8 Ozanne SE, Dorling MW, Wang CL, Nave BT. Impaired PI 3-kinase activation in adipocytes from early growth-restricted male rats. Am J Physiol Endocrinol Metab 2001; 280: E534-E539.

9 Fernandez-Twinn DS, Wayman A, Ekizoglou S, Martin MS, Hales CN, Ozanne SE. Maternal protein restriction leads to hyperinsulinemia and reduced insulin-signaling protein expression in 21-mo-old female rat offspring. Am J Physiol Regul Integr Comp Physiol 2005; 288: R368-R373.

10 Ozanne SE, Olsen GS, Hansen LL, et al. Early growth restriction leads to down regulation of protein kinase $\mathrm{C}$ zeta and insulin resistance in skeletal muscle. J Endocrinol 2003; 177: 235-241.

11 Obici S, Zhang BB, Karkanias G, Rossetti L. Hypothalamic insulin signaling is required for inhibition of glucose production. Nat Med 2002; 8: 1376-1382.

12 Spanswick D, Smith MA, Mirshamsi S, Routh VH, Ashford ML. Insulin activates ATP-sensitive $\mathrm{K}^{+}$channels in hypothalamic neurons of lean, but not obese rats. Nat Neurosci 2000; 3: 757-758.

13 Pocai A, Obici S, Schwartz GJ, Rossetti L. A brain-liver circuit regulates glucose homeostasis. Cell Metab 2005; 1: 53-61.

14 Aguilar-Bryan L, Bryan J. Molecular biology of adenosine triphosphatesensitive potassium channels. Endocr Rev 1999; 20: 101-135.

15 Sharma N, Crane A, Gonzalez G, Bryan J, Aguilar-Bryan L. Familial hyperinsulinism and pancreatic beta-cell ATP-sensitive potassium channels. Kidney Int 2000; 57: 803-808.

16 Livak KJ, Schmittgen TD. Analysis of relative gene expression data using real-time quantitative PCR and the 2(-Delta Delta C(T)) Method. Methods 2001; 25: 402-408.

17 Towbin H, Staehelin T, Gordon J. Electrophoretic transfer of proteins from polyacrylamide gels to nitrocellulose sheets: procedure and some applications. Proc Natl Acad Sci USA 1979; 76: 4350-4354.

18 Dahri S, Snoeck A, Reusens-Billen B, Remacle C, Hoet JJ. Islet function in offspring of mothers on low-protein diet during gestation. Diabetes 1991; 40: $115-120$.

19 Nieto-Diaz A, Villar J, Matorras-Weinig R, Valenzuela-Ruìz P. Intrauterine growth retardation at term: association between anthropometric and endocrine parameters. Acta Obstet Gynecol Scand 1996; 75: 127-131.

20 Devaskar SU, Thamotharan M. Metabolic programming in the pathogenesis of insulin resistance. Rev Endocr Metab Disord 2007; 8: 105-113.

21 White MF. Insulin signaling in health and disease. Science 2003; 302: 17101711 .
22 Barthel A, Schmoll D. Novel concepts in insulin regulation of hepatic gluconeogenesis. Am J Physiol Endocrinol Metab 2003; 285: E685-E692.

23 Oak SA, Tran C, Pan G, Thamotharan M, Devaskar SU. Perturbed skeletal muscle insulin signaling in the adult female intrauterine growth-restricted rat. Am J Physiol Endocrinol Metab 2006; 290: E1321-E1330.

24 Thorn SR, Regnault TR, Brown LD, et al. Intrauterine growth restriction increases fetal hepatic gluconeogenic capacity and reduces messenger ribonucleic acid translation initiation and nutrient sensing in fetal liver and skeletal muscle. Endocrinology 2009; 150: 3021-3030.

25 Jaquet D, Vidal H, Hankard R, Czernichow P, Levy-Marchal C. Impaired regulation of glucose transporter 4 gene expression in insulin resistance associated with in utero undernutrition. J Clin Endocrinol Metab 2001; 86: 3266-3271.

26 Ozanne SE, Jensen CB, Tingey KJ, et al. Decreased protein levels of key insulin signalling molecules in adipose tissue from young men with a low birthweight: potential link to increased risk of diabetes? Diabetologia 2006; 49: 2993-2999.

27 Niswender KD, Morrison CD, Clegg DJ, et al. Insulin activation of phosphatidylinositol 3-kinase in the hypothalamic arcuate nucleus: a key mediator of insulin-induced anorexia. Diabetes 2003; 52: 227-231.

28 Shyng SL, Nichols CG. Membrane phospholipid control of nucleotide sensitivity of KATP channels. Science 1998 ; 282: 1138-1141.

29 Trapp S, Ballanyi K. KatP channel mediation of anoxia-induced outward current in rat dorsal vagal neurons in vitro. J Physiol 1995; 487: 37-50.

30 Zawar C, Plant TD, Schirra C, Konnerth A, Neumcke B. Cell-type specific expression of ATP-sensitive potassium channels in the rat hippocampus. $J$ Physiol 1999; 514: 327-341.

31 Obici S, Feng Z, Karkanias G, Baskin DG, Rossetti L. Decreasing hypothalamic insulin receptors causes hyperphagia and insulin resistance in rats. Nat Neurosci 2002; 5: 566-572.

32 Pocai A, Lam TK, Gutierrez-Juarez R, et al. Hypothalamic KATP channels control hepatic glucose production. Nature 2005; 434: 1026-1031.

33 Novitskaya T, Baserga M, de Caestecker MP. Organ-specific defects in insulin-like growth factor and insulin receptor signaling in late gestational asymmetric intrauterine growth restriction in Cited 1 mutant mice. Endocrinology 2011; 152: 2503-2516.

34 Seghers V, Nakazaki M, DeMayo F, Aguilar-Bryan L, Bryan J. Sur1 knockout mice. A model for KATP channel-independent regulation of insulin secretion. J Biol Chem 2000; 275: 9270-9277.

35 Lane RH, MacLennan NK, Hsu JL, Janke SM, Pham TD. Increased hepatic peroxisome proliferator-activated receptor-gamma coactivator-1 gene expression in a rat model of intrauterine growth retardation and subsequent insulin resistance. Endocrinology 2002; 143: 2486-2490.

36 Sun Y, Liu S, Ferguson S, et al. Phosphoenolpyruvate carboxykinase overexpression selectively attenuates insulin signaling and hepatic insulin sensitivity in transgenic mice. J Biol Chem 2002; 277: 23301-23307.

37 Air EL, Strowski MZ, Benoit SC, et al. Small molecule insulin mimetics reduce food intake and body weight and prevent development of obesity. Nat Med 2002; 8: 179-183.

38 Carvalheira JB, Ribeiro EB, Araujo EP, et al. Selective impairment of insulin signalling in the hypothalamus of obese Zucker rats. Diabetologia 2003; 46: 1629-1640.

39 Coupé B, Grit I, Darmaun D, Parnet P. The timing of "catch-up growth" affects metabolism and appetite regulation in male rats born with intrauterine growth restriction. Am J Physiol Regul Integr Comp Physiol 2009; 297: R813-R824. 